\title{
Designing for Social and Physical Interaction in Exertion Games
}

\author{
Florian 'Floyd' Mueller, Martin R. Gibbs and Frank Vetere
}

\begin{abstract}
Exertion games lend themselves to facilitating social and physical interactions, in particular when compared to button-press games. However, there is little understanding of how specific aspects of an exertion game's design can facilitate these social and physical interactions. In response, we present a set of design themes based on our analysis of players' experiences with a distributed table tennis game. The themes are: Shared Object, Anticipation, Secondary Performance, Movement Variety, and Uncertainty. We hope that these themes can guide other designers who aim to support social and physical interaction in order to support players profiting from the many associated benefits.
\end{abstract}

Keywords Exertion games - Social interaction - Physical interaction - Design • Shared objects

\section{Introduction}

Over the last decades, user interface research on social and physical interaction has gained an increased focus in the field of human-computer interaction (Dourish 2001). In particular, designers and researchers are interested in developing playful experiences that support social and physical interactions and promise to be "fun" (Bekker et al. 2010). In this chapter, we focus on playful interactions that involve more than one person interacting and that support physical engagement with the

\author{
F. F. Mueller ( $\bowtie)$ \\ Exertion Games Lab, RMIT University, Melbourne, VIC, Australia \\ e-mail: floyd@exertiongameslab.org \\ M. R. Gibbs · F. Vetere \\ Interaction Design Lab, The University of Melbourne, Melbourne, VIC, Australia \\ e-mail: martin.gibbs@unimelb.edu.au \\ F. Vetere \\ e-mail: f.vetere@unimelb.edu.au
}


environment. The physical interaction borrows from tangible computing (Hornecker and Buur 2006) as well as physical exertion. Exertion interactions are interactions that require intense physical effort from players (Mueller et al. 2003).

In the past, several attempts have been made to understand the relationship between the interface and social and physical experiences (Lindley et al. 2008; Mueller et al. 2011; Bekker et al. 2010), however, there still exists only a limited knowledge on how to design for social and physical exertion play.

In order to contribute this knowledge, we have created and studied a novel exertion game based on table tennis, which resulted in several contributions around our analysis of the design (Mueller et al. 2008b, 2009, 2010). We now put forward a set of themes that we have derived from the analysis of player interactions with this game, which we believe can be useful for designers of user interfaces that aim to support social and physical interaction.

The presented case study is of a physical game for three distributed players called "Table Tennis for Three." The game is loosely based on table tennis. However, it offers a gameplay experience through virtual augmentation, which is quite different to traditional table tennis. The virtual augmentation utilizes a videoconferencing component to allow for distributed play while the affordances of the bat and ball support movements that aim to maintain the benefits of physical play. This mixed approach can offer novel user experiences such as supporting geographically distributed participants. The game also supports three players at the same time equally, inspired by the opportunity of networked games to support large user numbers. We note that, first, supporting three players is not easily achieved in traditional table tennis (where it is often two against one). Second, although we acknowledge that three players is not comparable to the large number of players supported by many networked games, we see this as a first step in exploring the scaling of numbers in exertion games.

\section{Related Work}

A heightened consideration of social and physical aspects in digital play has a history in human-computer interaction with a focus on social and bodily interactions (Dourish 2001). Going back even further, we can see how Merleau-Ponty proposes a perspective highlighting how the human body is mutually engaged with the mind and that an intertwined connection exists helping us interact with our environment and other embodied beings. He argues that we need to consider our bodies and how we interact with other bodies if we want to understand what it means to be human (Merleau-Ponty 1945).

Researchers in human-computer interaction similarly stress the importance of the human body when it comes to the digital world, such as Winograd et al. who argue for a more nuanced view on the embodied user when interacting with computers (Winograd and Flores 1986). Dourish identified an embodied focus in the tangible and social computing systems developed around him (Dourish 2001), 
arguing that embodied features of interactive systems are related to the features of social settings. These high-level investigations are often based on earlier philosophical arguments made by Merleau-Ponty (Merleau-Ponty 1945), which are then applied to interactions with computers. Many of these arguments highlight the role of play in social and physical interactions. This role of play we will investigate in more detail next.

Lindley et al. suggest that a game's nature can change when players are involved in full bodily actions, rather than button presses, from a traditional virtual game experience of "hard fun" to more social play (2008). Similarly, de Kort et al. promote the consideration of full body actions because they believe players have an intrinsic need to experience their physical environment kinesthetically. The authors argue for a relationship between physical environment and virtual gameplay and propose "sociality characteristics" for games, which include a consideration of exertion actions, as they can "radically" impact social play (de Kort and IJsselsteijn 2008). Moen (2006) highlights the role of free-form movements and presents a framework for kinesthetic movement interaction, arguing that technological augmentation can support novel experiences. However, the author concentrates on single-user interactions, only recently looking at social play (Segura et al. 2013).

There have been several design-oriented explorations on the topic of social and physical interaction in exertion games from which we learn. We articulate these next.

Fish'N'Steps is a social approach to combining physical bodily actions with virtual content to enhance healthy activity. It is a mobile application that is aimed at encouraging participation in walking activities via social comparison (Lin et al. 2006). The proposed system works in combination with a pedometer to motivate an increase in a participant's daily energy expenditure. It separates the physical activity from the social activity; only after the participants walked all day can they share their progress through a fish tank metaphor displayed on a screen in an office. Their bodies rest while they assess their relative progress.

Consolvo et al. present another distributed pedometer-based system implemented on a mobile phone (Consolvo et al. 2006, 2008). The authors identified design requirements that include aspects regarding the body, the users' environment, and virtual augmentation. Systems that combine real-world physical exertion with virtual aspects of a videoconference have suggested that there might be the possibility for social facilitation. For example, exercise bikes have been networked to allow for distributed races in a competitive environment. The bodily component suggests the use of physiological data to enhance the distributed experience, as participants reported that visualized heart rate from a remote rider could motivate them to cycle faster (Bikeboard). The presence of a remote participant appears to affect the exertion performance; however, in exercise bike cycling the participants cannot interfere with one another physically. In many team sports, players can actively prevent their opponents from achieving the game's goals (Vossen 2004). This aspect of the shared experience is missing in networked bike riding; however, it has been explored in an early game of ours, Breakout for 
Two (Mueller et al. 2003). Breakout for Two is a synchronous exertion game for two players with an integrated video communication channel. An evaluation showed that players were able to form a social bond with each other despite the geographical distance between them. However, what design aspects contributed to the social play and what role the physical actions as well as the physical environment, such as the two-location setup, had on the resulting experience remain unexplored. Besides Breakout for Two, there is a shortage of projects that look into exertion experiences where participants can interfere with each other. We are interested in table tennis, which is such a game where participants interfere with each other, hence we are looking into table tennis projects next.

The game of table tennis has inspired other research projects (Ishii et al. 1999; Knoerlein et al. 2007; Lawn and Takeda 1998; Woodward et al. 2004). Most of these implementations focus on the demonstration of the technologies' capabilities, showcasing how mechanical and computational advances can simulate certain aspects of table tennis, for example force-feedback (Knoerlein et al. 2007). The outcomes of these projects suggest that simulating and recreating a traditional bodily game such as table tennis over a computer network is probably still not technically feasible and costly.

In summary, previous research suggests that the consideration of the human body and its physically effortful interactions in interactive systems can facilitate beneficial experiences. Most utilized approaches are based on an embodied perspective that highlights the importance of the users' body being situated in a physical environment, interacting with other beings. However, there is a limited understanding of what role design plays when bodily actions are augmented with technology. The approach taken in this work is to suggest an interrelationship between social and physical exertion play and this article examines how the game's design can facilitate this interrelationship to provide a benefit to the experience. This approach is explored through a case study of a prototypal system called "Table Tennis for Three." This case study highlights the potential for novel experiences such as unique social support in terms of number and geographical location of participants, difficult to achieve without technological augmentation. Investigating this can contribute toward an understanding of the role of design in supporting social and physical exertion play, which in turn can support the leveraging of the many benefits physical activity affords, guiding future advances in this field.

\section{Table Tennis for Three}

We present the analysis of a study of Table Tennis for Three. It shows how social and physical exertion play can be supported and facilitated by certain aspects of the design and articulates what these aspects are, as expressed through a set of design themes. The intention is that these themes are to be used by designers who aim to support social and physical exertion play in future interfaces. 


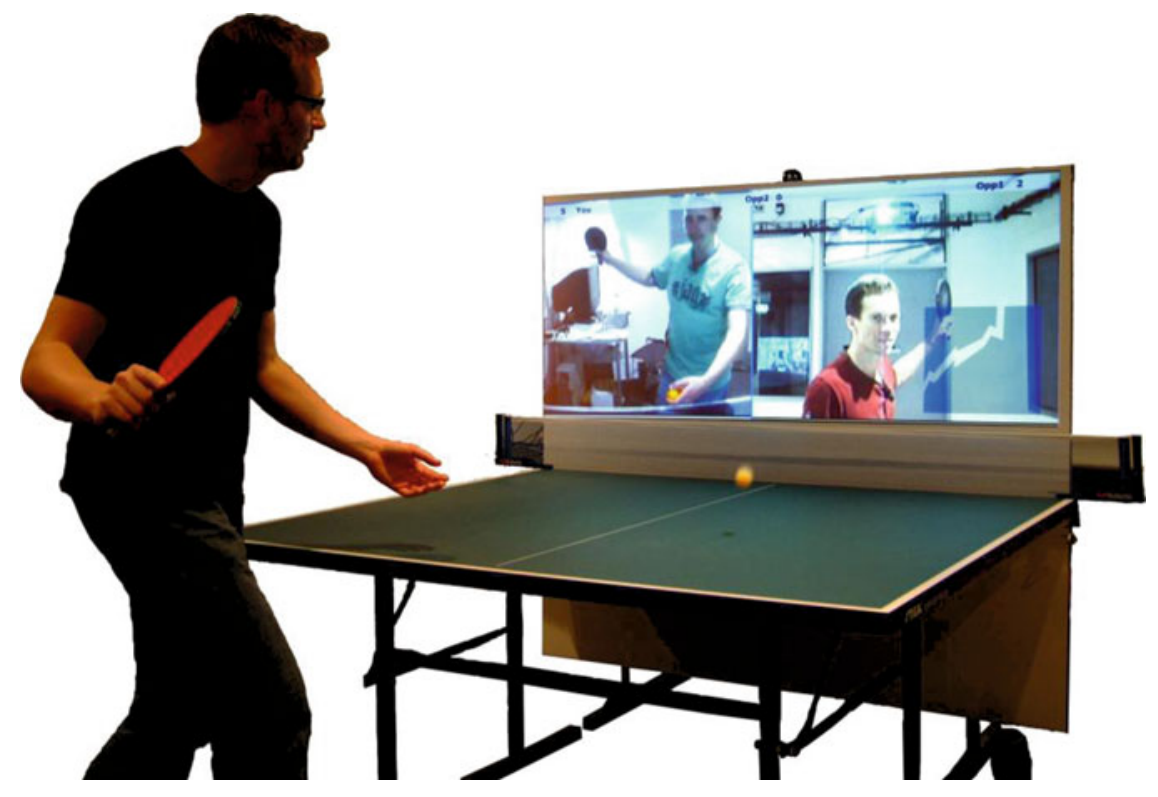

Fig. 1 Table tennis for three

\subsection{Playing Table Tennis for Three}

We now describe Table Tennis for Three, an exertion game that uses a physical ball, bat, and table for play and supports players from three geographically distant locations. In particular, this prototypal system highlights two opportunities of technology to support playful experiences that are otherwise difficult to achieve without technology. First is the opportunity to support geographically separate players, playing together simultaneously (in contrast to taking turns). Second is the opportunity to scale the play experience, such as allowing three participants to play together equally.

\subsubsection{The Setup}

Each player uses a physical ball, a bat, and a table tennis table. The table is set up in such a way that the ball can be hit against the vertically positioned opposite half of the table (Fig. 1). This setup is familiar to table tennis players who practice alone by playing the ball against the board. This backboard has projected images of eight large "bricks" on it. These bricks are identical for all players, and they are synchronized across all three stations (Fig. 2). A projector mounted to the ceiling projects the bricks in a semi-transparent fashion on top of two video streams of the other players in the game. One player's videoconference is positioned on the left of the backboard, and the other is on the right. Each table has a set of loudspeakers 

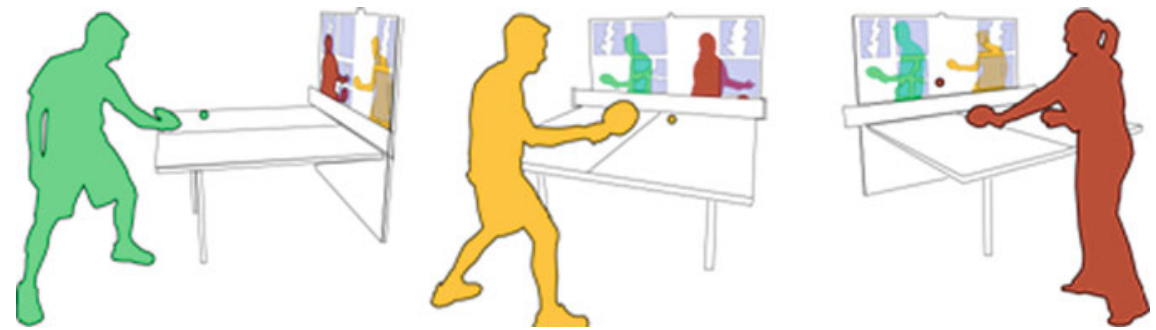

Fig. 2 The bricks are shared across the three stations, a hit is visible to all players

and each player wears a microphone so the three participants can converse with each other in a three-way audio conference during the gameplay.

The backboard is equipped with sensors mounted on the back that detect when and which brick the players hit. These piezoelectric sensors detect the impact of the ball hitting the wooden table tennis table, with the impact permeating through the wood. The sensors are located in a manner similar to the one suggested by Ishii et al. (1999) in order to determine the exact impact location based on the time it takes the impact to travel to each sensor: the sensor that receives the impact first is triggering the impact location to the software system using a digital acquisition board that samples the impact data at a high-speed.

The bricks "break" when hit by the ball as a result of the sensors registering the location of the impact. All three players see the same brick layout and the same brick status layered on top of the videoconferencing streams. If a brick is hit once, it cracks a little. If it is hit again (regardless of by which player), it cracks more. The crack appears on all three stations. If hit three times, the brick "breaks" and is removed from play, revealing more of the underlying videoconference: the player "broke" through to the remote player. However, only the player that hits the brick the third and final time receives the point. This offers players a number of strategies for winning the game. The players can either try to crack as many bricks as possible by placing the ball quickly or they can wait for the opportunity to snatch away points from other players through hitting bricks that have been already hit twice by the others.

Each brick that is completely broken scores one point, and the running score is displayed along the top end of the projection. Play continues until all bricks have been cracked three times and been removed from play. At this point the player who has scored the most points is announced as the winner and after a delay of $15 \mathrm{~s}$, the game resets all the bricks and play can recommence.

\subsection{Studying Playing Table Tennis for Three}

The data used for the analysis of playing Table Tennis for Three comes from video recordings of participants playing Table Tennis for Three and videotaped interviews of all three participants together in one room. Notes were also taken during 
the interviews. The interviews contained open-ended questions about the players' experiences and their interactions with one another. Furthermore, we asked participants to answer a short questionnaire to examine specific questions we had on experience and gameplay (more on this later).

We recorded the participants during play with a video camera. Only one player was recorded at a time. If notable actions occurred on the remote end, this was observed through the videoconference. The interviews were also videotaped.

Each group played between 30-60 min. The players were brought together into one room after the game, where the interviews were conducted with all three of them together. The interviews lasted from 20 to $60 \mathrm{~min}$.

\subsubsection{Participants}

We recruited 42 participants through personal contacts, email lists, and word-ofmouth. The volunteers were asked in the advertising material to organize themselves preferably in teams of three. If they were unable to do so, we matched them up randomly with other participants in order to have always three people participating at the same time.

We report on 14 teams of three. One participant played twice due to a lastminute cancellation (which was considered in the analysis). In total there were therefore 41 participants. The participants were between 21 and 55 years old (mean 31.6 years), and consisted of 27 males and 14 females. It is acknowledged that prior social relationships between participants can affect the social play interactions within a game (Salen and Zimmerman 2003), this aspect was taken into account by asking additional questions about how their existing relationships affected the way they played. The participants' prior exposure to table tennis was varied. One had never played before, 14 participants played less than five times, 18 players between five and 100 times and eight volunteers played more than 100 times before.

\subsubsection{Data Analysis}

The video data was analyzed by the authors using an iterative coding process, drawing on sensitizing ideas that relate to the research question and the relevant literature. The coding process was used to identify important concepts. The data was coded to locate concepts in an attempt to condense the data into categories. We created annotations on segments of the data we found particularly interesting, and wrote down memos during the coding process as reflective commentaries for deeper analysis.

The list of concepts we created from the first pass helped identifying emerging themes, and informed the next coding step when we viewed the data again. In this second iteration, the focus was more on the concepts and the initial codes were reviewed and examined before checked if some of them could be combined. 
In a subsequent viewing of the data, the focus was on instances that illustrated concepts. We tried to make comparisons and identify contrasts to support the conceptual coding categories we have developed so far. Then, the emerged themes were grouped into logical groups and hierarchical categories were created. We then sorted the annotations, memos, and codes into groups to identify the key themes, looking out for whether they would be specific for Table Tennis for Three or exertion games in general.

\section{Results}

We begin by providing evidence that both social and physical exertion play occurred when engaging with Table Tennis for Three. What follows is a description of themes identified from the data.

\subsection{Social Play}

The players encouraged themselves and each other during the game.

Lets' go for it! [P3, play]

$\mathrm{P} 18$, come on! [P20, play]

They were not shy of engaging in competition, often expressed in statements such as:

I really wanted to beat her. [P13, interview]

If a player snatched away the last brick, there were statements such as

You $\mathrm{b}^{* * *}$ ! [P21, play]

Although players interacted verbally with their partners during the game, some players also commented that they felt more like they were playing against bricks:

[...] not at each other. When playing, you are more focusing on the blocks than on the other player. [P15, interview]

P21 described vividly how she recognized that she could have focused on the bricks, but realized that playing against "a person" suited her better. In the interviews she made the following comment to the third player P4 in her group:

[...] you are playing against yourself, sometimes, because you are very competitive against the screen, whereas I was watching P13, and it was like, when I was telling her off for cheating, then I actually aimed the ball at P13's head, at the screen! (All laughing). 


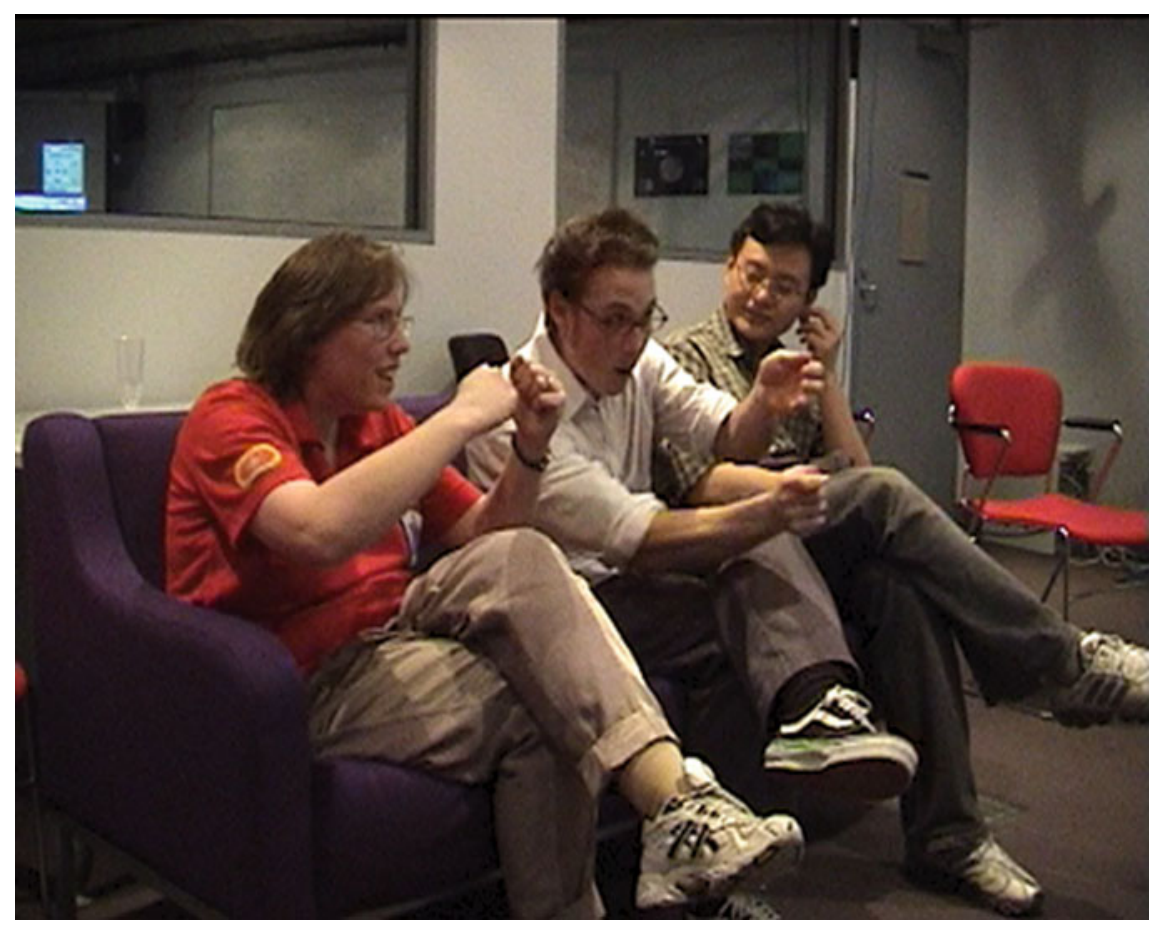

Fig. 3 During the interview: “... and you just wait for the third one [crack] and try to get in there quick!"

\subsection{Exertion Play}

Participants were investing physical effort, and as a result, were exhausted. They commented on how quickly the game made them tired, in particular because they never had to wait for their partner, as in conventional table tennis. Participants also showed visible signs of exertion, such as sweat on their foreheads.

Next, an examination follows that investigates what aspects of the design facilitated both social and physical exertion play.

\subsection{Movements Responding to Partners' Movements}

The participants aimed at hitting bricks that have been hit before (see Fig. 3):

And you just wait for the third one [crack] and try to get in there quick (makes a smash arm movement) [P11, interview]

[You] wait for someone to break the blocks and go for it [P10, interview]. 


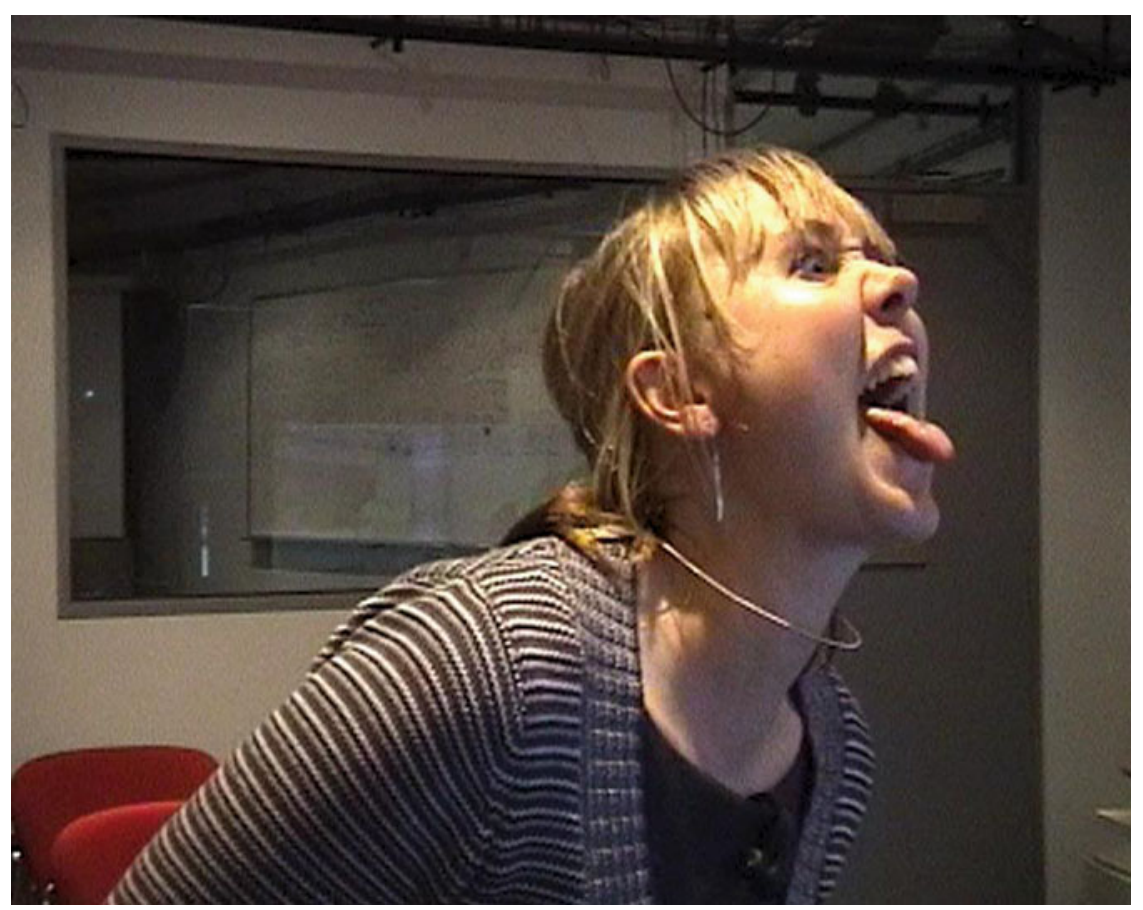

Fig. 4 Player's reaction from playing against another player

However, their ability to anticipate which brick will be hit next was limited:

[There is a limited] sense where the other player is [...] [or] where he is playing [P12, interview]

[...] so I didn't know this was where you were throwing the ball [P13, interview]

Anticipating the other players' movements mostly only played a role in initiating the game. P14 said during the interview:

"You are waiting, and as soon as you are ready" [both P14 and P1 initiate a gesture for a serve but stop just before executing it], P1 interrupts: "You are ready to go" [both finishing their movement, both smiling].

\subsection{Being Expressive}

Users demonstrated affective expressions during play, but predominantly outside immediate play: in between points, while having a break, and after the games (see Fig. 4). Most verbal interactions were of emotional nature and not in relation to play directly, such as swearing, yelling, or dismissing the other player, but sometimes also words of encouragement were exchanged: 
You are not trying hard enough! [P5, play],

I'm going to beat you next time [P7, play], and

You guys are hysterical! [P8, play].

Most of these verbal exchanges were of a joking nature, with elements of mocking, teasing or "fooling around," characteristic of social sports games (Weinberg and Gould 2006).

Some users chose to supplement their verbal comments with a gesture, such as throwing their hands in the air to indicate they won. A player jokingly made a fist to the other players; another participant put her tongue out. Players often applauded others on their performance, and some performed little winning dances. This was often accompanied by laughter, facilitating a humorous atmosphere.

The exertion activity served as a starting point for social interaction, but it also hindered social interaction when attempted verbally: Players sometimes wanted to say something, but were not able to because they were too exhausted or too involved in their exertion activity. A player made this explicit by saying

hang on... [P5, play]

when the beginning of a new game interrupted his dialog. He continued his verbal exchange in the next break of the game after he recovered from his exhaustion.

\subsection{Alternative Ways of Playing}

Players adapted rules from traditional table tennis to suit the interactions afforded by Table Tennis for Three. They also referred to table tennis and its rules in the interviews. Even though none of the teams discussed rules amongst each other before the game, they successfully engaged in gameplay by assuming the ball needs to be hit with the bat, cannot be returned volley, etc.

Nevertheless, players changed the way they played when an opportunity arose, such as when a player could not return the ball with his/her bat, but was able to catch it with her/his hand to increase the chance of winning. They also grabbed spare balls and played them successively, and hid under the table before serving (see Fig. 5).

The players knew that this was "breaking" the rules, because they pointed it out if they caught someone: a player yelled out loud

You cheat, you are a cheater! (accompanied by laughter) [P9, play]

because a remote player was using her hands, but then this player used the hands herself. 


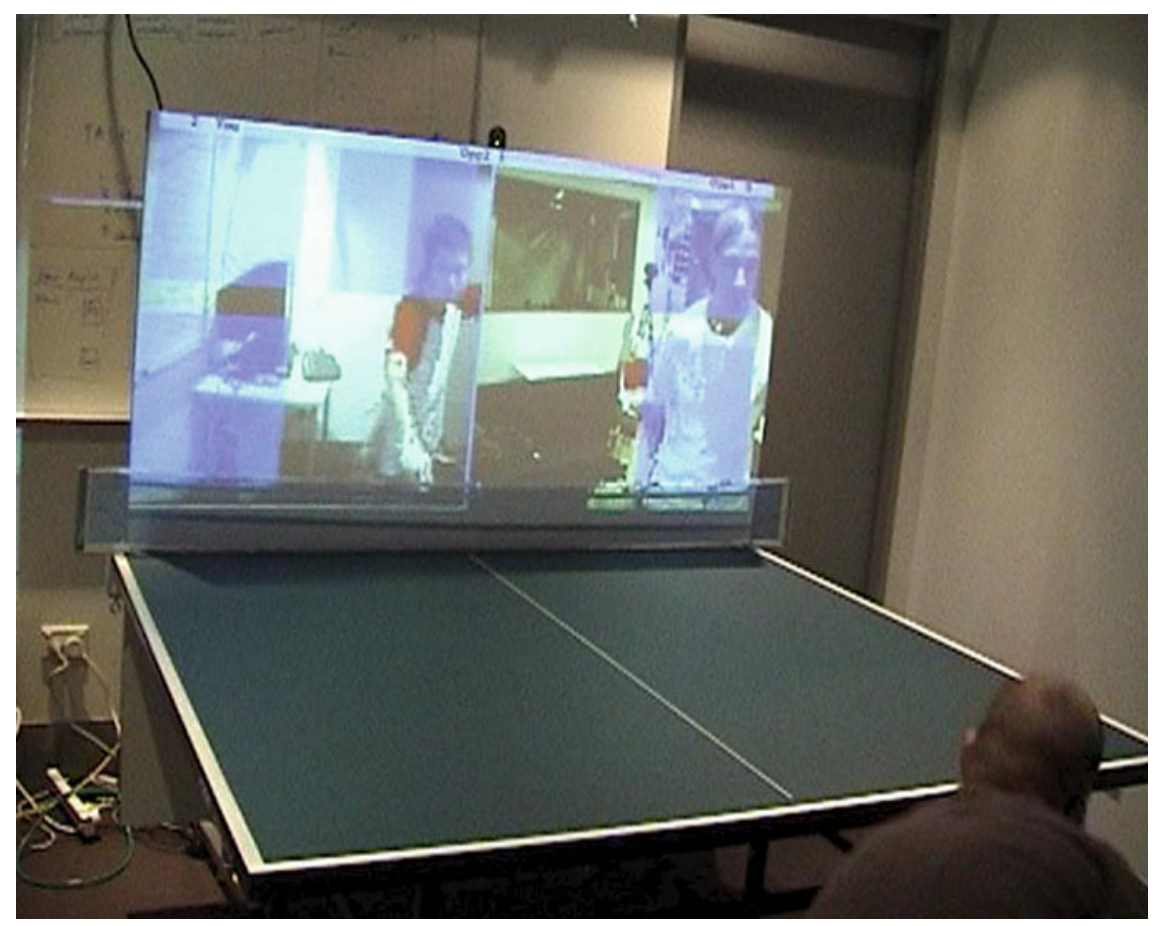

Fig. 5 Player hiding to try alternative serve

\subsection{Unpredictability of Physical Ball}

Despite the players' best efforts, the ball often acted in unexpected ways; it hit the edge of the bat, and was diverted in the opposite direction. The ball also often bounced back quicker than expected, but a quick reaction on impulse of the player was able to deflect the ball in a manner that resulted in an unanticipated trajectory of the ball. The ball also often hit the edge of the table, being reflected off it in a surprising angle. Players looked amazed at how some of their hits returned the ball. These surprising situations are characterized by a considerably unexpected behavior of the ball that the player with his/her actions did not intend nor anticipated.

The challenging aspect of controlling the ball with the bat and the associated surprising actions that occurred contributed to the players' enjoyment, which was reflected in their verbal expressions: players often shouted short exclamations such as

yikes!

when the ball flew off in the wrong direction, often followed by a smile (see Fig. 6). This laughter was then answered with laughs by the remote players, and 


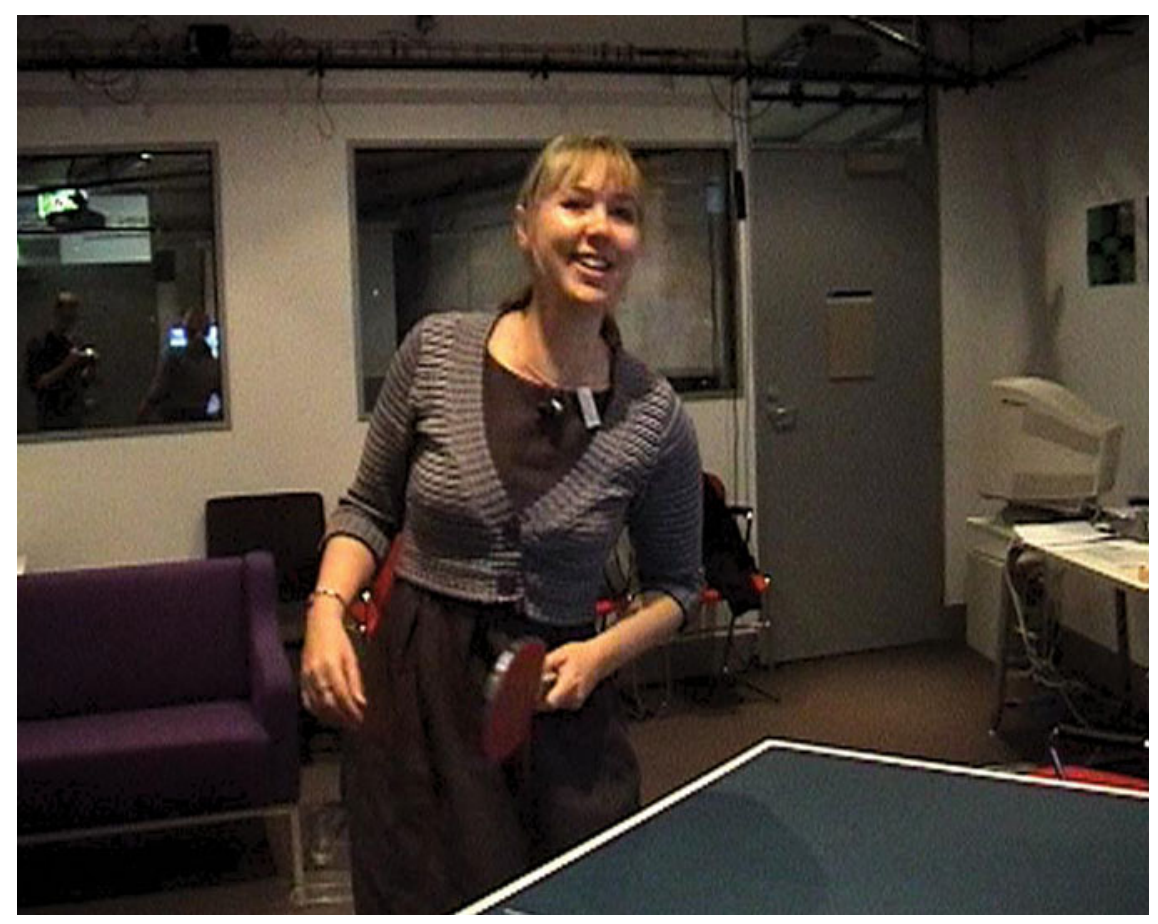

Fig. 6 Ball flew off the side

functioned as conversation starters. Players then switched their attention to the remote end if they heard such a surprise expression.

\section{Discussion}

We now discuss the observations in regards to social and physical exertion play and associated themes that facilitated this social and physical exertion play.

\subsection{Shared Virtual Objects}

On the one hand, participants appreciated being able to play with remote partners. They showed signs of playing together despite the fact that interactions were mediated. On the other hand, players reported that they sometimes felt more like playing against the virtual bricks rather than another person. We also observed incidences where players seemed to experience both, playing against another person and playing against bricks, such as described by the group around P21: they seemed to be able to navigate between these apparently contradicting experiences. 


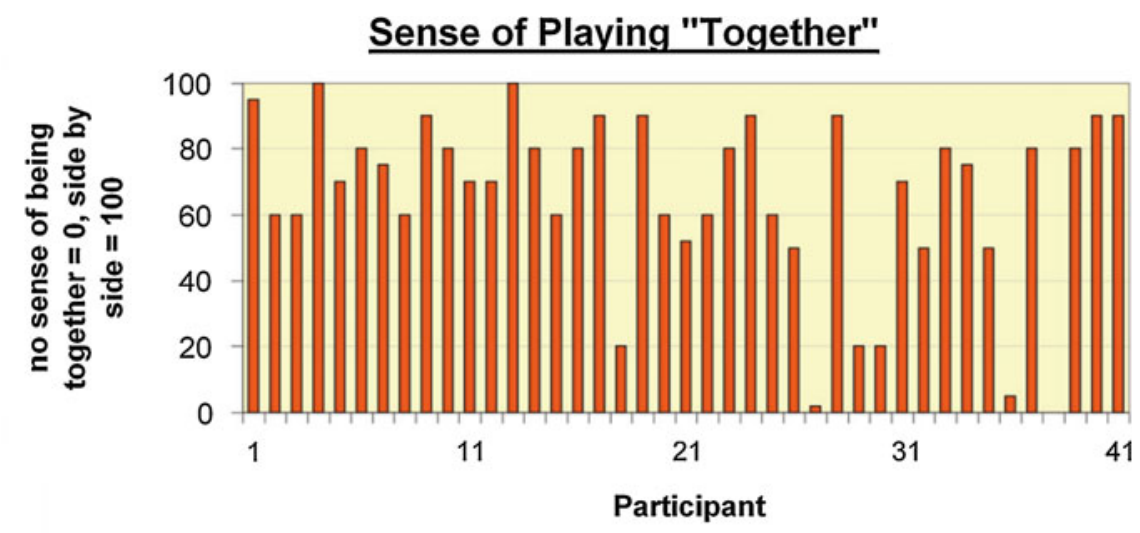

How strong was your sense of playing together, compared to playing on the same table together

Fig. 7 Sense of playing "together" rated

We asked the participants to rate their sense of having played together on a scale from 0 to 100 , with 0 being no sense of playing together, and 100 being the same extent as when playing on the same table.

The median was 70, and the arithmetic mean 65.46 (see Fig. 7). The answers were affirmative, especially in combination with the fact that most players ( 35 out of 40) had the feeling they were doing something together. Interestingly, this was statistically significant if correlated with "I liked the game" $(r=0.64, p<0.01)$. The players who disagreed with the "together" statement were among the three that disliked the game, suggesting that there could be a link between the engagement with the game and the feeling of "doing something together."

In order to understand this further, we look at the idea of parallel and nonparallel play (Mueller et al. 2008a): by parallel play, we mean play experiences in which participants are aware of each other's exertion activity, but are not physically interfering with one another. A typical example from traditional sports is a 100-m race, in which the white lines on the track explicitly forbid any physical interference between runners. Vossen (2004) says that in parallel play, each player performs his or her exertion actions independently from one another. The players have no direct influence upon the difficulty of the task faced by other players, as they cannot directly interfere with one another.

By non-parallel play we mean play experiences in which participants can use their bodies to physically interfere with one another. An example from the traditional sports domain is wrestling, where the wrestlers contend for control of each other's bodies. Vossen (2004) reminds us that this category is distinctly different to how people can experience exertion compared to parallel play.

Table Tennis for Three facilitated parallel play in the physical world of the exertion actions, but also non-parallel play in the virtual world of the shared 
bricks. This explains why players expressed both, that they felt like playing against bricks but also against other players. The virtual bricks enabled a non-parallel game activity, which the players used to challenge each other in order to enrich their social experience. The players used this opportunity to engage with their remote opponents, trying to "outsmart" them to snatch points using tactical decisions to gain an advantage. As such, the virtual bricks functioned as "shared objects" that facilitated the non-parallel play. Shared objects are common in many traditional social sports; often it is a ball, such as in conventional table tennis. In Table Tennis for Three, the virtual bricks took on the role of shared object, as the physical ball was unique to each location and hence not shared.

The main exertion action of the game, however, is the hitting action of the ball, which occurs in the physical space and is of a parallel characteristic. This parallel characteristic facilitated a sensation of playing against bricks, not other players, affecting social play. The players described the parallel aspect through expressing they felt like "racing" the computer bricks. When players had such an experience of "playing against bricks" and "not at each other," the mediating design fell short in facilitating a non-parallel experience at that moment.

The affordances of both parallel and non-parallel play were used by some players to enhance their experience. Especially, the players who seemed to seamlessly navigate between playing against another person and against virtual bricks demonstrated that the boundaries of the spaces can become fluid in the context of use, and the users made them fit to meet their interactional needs.

It is also interesting to note that due to limitations in the sensor and network technologies, delays in brick updates (changing from a full brick, to a broken brick, to a disappearing brick) can occur. Network lags in online games can significantly affect the user experience (Linehan et al. 2006), and are a challenge for any online experience. Interestingly, as the physical ball required skillful control by the participants, it appeared this focused their attention on the exertion actions rather than the updates of the virtual bricks, which in turn might limit any negative effects delays in brick updates might produce. As such, any effects resulting from delays in Table Tennis for Three appeared to be tempered by the focus on the exertion actions, especially when compared to button-press computer games.

In sum, players experienced both social and physical exertion play, even though the interaction between the participants was mediated by technology. Interestingly, the social play comprised of both parallel and non-parallel play, where parallel play was occurring in the physical space, and non-parallel play in the virtual thanks to shared virtual objects. Players were able to navigate both spaces and both forms of social play, which was facilitated by the physical exertion play activity.

We now present a summary of the result and discussion, phrased in an actionable way to guide designers of future systems:

Shared virtual objects can be used to support social play. However, they might facilitate non-parallel play in the virtual world, whereas the exertion activity can be parallel in the physical world. 


\subsection{Anticipation}

A key characteristic of exertion games is the involvement of bodily movements, and supporting bodily movements is believed to facilitate social interactions (Lindley et al. 2008). For individual activity, this support of bodily movements is often associated with self-awareness of exertion actions (Moen 2006; Consolvo et al. 2006), however, in a social setting, awareness of other bodies and their associated movements becomes also important (Fogtmann et al. 2008): an understanding of the activities of others can provide a context for one's own activities (Rettie 2003), and so-called awareness of other bodies can be an important part of how we make sense of the world through our bodies (Dourish 2001).

Knowing that awareness is an important element in supporting social play, it can now be discussed how anticipation was facilitated by the videoconferencing component. Players were contending for control of the virtual bricks. In order to gain an advantage, participants tried to anticipate their partners' next moves. Such anticipation was possible because movement was involved, and this movement was continuous. Participants could perceive this continuousness of movement through the videoconference.

The notion of continuousness draws attention to the fact that bodily gross-motor movements, as featured extensively in Table Tennis for Three, are accompanied by preparatory and follow-through movements, quite different to button presses. An exertion action involves movements that include a whole set of motions (Moen 2006): for the Table Tennis for Three players, it was a backswing, a forward swing, the contact with the table tennis ball, and the follow-through. Although only the contact with the ball counted toward the game, all motions formed part of play, and players tried to make use of it. Players were aiming to be aware of their opponents' bodily movements by attempting to "read" these pre- and postmovements to anticipate future play actions. In Table Tennis for Three, the participants made use of the continuousness nature of bodily movements in order to enhance their social play experience through anticipation.

When compared to traditional gamepad-controlled games, it can be seen that continuousness has a particular role in exertion games. Being aware of a player controlling a binary button interaction does not reveal much of that player's intention behind the button pressing action. Anticipating a future move is very difficult with buttons, as they do not require visible sequential movements (a simple flick with the thumb is sufficient) that participants could use as cues to initiate an action. Usually, this support for continuousness is assumed in co-located experiences, however, the mediation in Table Tennis for Three brought this point to the fore, as the videoconference's technical shortcomings hindered the opportunities for anticipation.

Continuousness is a direct result of "movements [being] situated in time and space" (Griffin 2005). That is why animations representing player actions in computer games such as fighting games display pre- and post-movements in 
response to a single button-press. For example, a simple button-command in Tekken (Namco 2010) results in the character preparing for the kick by leaning backwards and moving the arm back, before propelling forward, hitting the opponent and then getting the arm back into the original position. As a consequence, if game designers want to include anticipation in conventional computer games, they need to support this anticipation by implementing it in the virtual world. In exertion games, players can utilize anticipation due to the continuousness characteristic of movement; this means that designers have an additional way to support anticipation.

Interesting to note is that players in Table Tennis for Three utilized both, the continuousness afforded by bodily movement as well as computer game play elements to anticipate future states of play. The players used the state of the bricks to anticipate which of them will be hit next and adjusted their actions accordingly. The players anticipated future actions not only based on movement actions over the videoconference, but also based on the movements in the virtual space.

Any mediating technology can affect how anticipation is supported in exertion games. In Table Tennis for Three, the capture area of the camera limited the space in which continuousness movements could have been captured and hence transmitted, and the videoconferencing quality failed to consider very fast movements and hence anticipation was limited. Also, the conical shape of the camera did not always adequately capture the ball's flight path, hindering any anticipation of future moves based on the location of the ball. However, anticipation was further supported through the virtual objects in the game. In contrast to sports (anticipation occurs based on continuousness in the physical space) and computer games (anticipation occurs based on actions in the virtual space), anticipation in Table Tennis for Three occurred based on continuousness over the videoconference as well as based on actions in the virtual space.

Even though Table Tennis for Three supported anticipation, it did not support bodily reciprocity as, for example, a conventional game of table tennis. Although players in Table Tennis for Three were anticipating their partners' next move, they were also dealing with the task of controlling their own ball, which was acting independent from any other player. In conventional table tennis, every return hit is a response to the other player. Players in Table Tennis for Three could choose to respond to the remote players, but they could also choose to engage in parallel play, just aiming to hit bricks in a random fashion as fast as possible. So the ball was not the mediating object between the players, but the bricks, where the ball was the mediator to the bricks. In addition, due to the conical shape of the videoconference, it was difficult to link partners' actions to specific bricks: although one could anticipate when a player is going to execute a hit due to the continuousness of movement, anticipating exactly which brick will be hit was difficult to anticipate once the ball left the capture area of the camera.

Supporting the continuousness of bodily movements allows anticipating a player's next action, contributing to social play. 


\subsection{Secondary Performance}

Participants performed using their bodies as a way to communicate, in particular outside the game, as a form of metagaming. Metagaming is a social play phenomenon that refers to the relationship of a game to elements outside of the game. One way that metagaming occurs "during a game other than the game itself $[\ldots]$ are social factors such as competition and camaraderie" (Salen and Zimmerman 2003). Our participants engaged in a metagame experience by socially interacting with one another beyond the immediate game play. In particular, through the exertion nature of the game, they did so by using their bodies to communicate: first, exhaustion made verbal exchange more difficult, second, the bodily focus of the game lent itself to communication behavior through the body. The participants embraced this by nonverbally commenting on other players' performances and turning the game into a bodily spectacle beyond the gameplay itself. Larssen et al. (2004) found the notion of "expressive latitude" particularly fitting in the context of exertion games to describe such behavior that is not directly influencing the game outcome, but can have communicative aspects. We call this secondary performance.

Interestingly, aspects of secondary performance also appeared in the interviews, in particular when participants were "retelling their experience." The retelling of what happened in a game is an important part of a "lived experience" (McCarthy and Wright 2004). Players predominantly used their exertion skills in the games, so they drew on these skills again during the reliving of the experience. This reliving of a "pleasurable kinaesthetic stimulation" can re-trigger the associated pleasurable emotions (Iso-Ahola and Hatfield 1986). Re-enacting the exertion movements can also support the player's cognitive processes, helping them remembering certain parts of the game (Lindley et al. 2008). Players gave further meaning to these exertion actions by reliving and sharing them with others, the support for secondary performance appeared to contribute toward a social play experience. These exertion actions supporting secondary performance are missing in conventional gamepad-controlled computer games, and their players have to rely on their cognitive skills to remember their lived experience and associated affective responses.

Supporting people in expressing themselves using their bodies-in and outside the game-can contribute to social play and facilitate metagaming.

\subsection{Movement Variety}

The participants exhibited bodily movement in many ways while striving to achieve their goals; we consider this a form of self-expression. The participants played with the limitations of technical mediation, using it as resource for "bending the rules." This cheating was used to enhance the experience, a 
phenomena which has been previously observed in traditional computer game play (Consalvo 2007). However, the inclusion of exertion in Table Tennis for Three afforded a new approach: players explored their bodily movements within the space of opportunities the sensing technology allowed (or not allowed, as not all actions were sensed), and what was communicated over the videoconference. Benford et al. used the terms "expected, sensed and desired" to differentiate different interactions within sensing systems (2005); using his words we could say that the participants were playing with the various categories that the sensing systems afforded. As the context was a game, the participants explored their movement variety within the sensing space in order to enhance their experience. The players were not so much trying to "break" the rules as to rather "bend" them, exploring alternative ways to achieve the goal in order to catch up with an advanced player, make the other player laugh, and so forth. This has been described as transformative social play, in which players actively engage with the rule system in order to shift or extend their relations with other players (Salen and Zimmerman 2003), therefore constituting a form of social play. The mediated aspect appeared to contribute to this, as it enabled a variety of opportunities for bending the rules.

The limitations of the technology enabled these opportunities in three ways:

1. The sensing system of the table tennis table afforded players exploring different ways to execute a hit: if I throw the ball, is the hit also registered? What if I throw it really hard, can I confuse the sensors so I break two targets? Players were asking these questions in the interviews but also trying out the different tactics during the game.

2. Players realized that they could grab another ball in their other hand to serve as backup if the first ball goes astray. This was difficult to see over the limited video quality, especially in fast-paced games, as the videoconference system communicated only a limited resolution displaying a limited framerate. Communicating subtle details in rapid actions, as often the case in exertion games, is still a challenge for sensing and networking technologies. Players are aware of this and they play with it to their gaming advantage.

3. There was a mismatch between the space the player occupied and what the videoconference camera saw. Participants played with this mismatch (for example by hiding below the table), contributing to a joyful atmosphere, but it also affected social play negatively. In co-located exertion games, the physical space and what the other player sees are usually interconnected. In distributed games, however, these spaces might be disparate due to technical limitations. In non-exertion games this mismatch might not be important, as players might not move much. In exertion games on the other hand, players' activities involve many large-scale movements, which might conflict with conventional awareness technologies that are aimed at supporting focused awareness cues such as facial expressions. When one of the participants stepped out of the view of the camera, she became "unsensable" by the camera. By doing so, she also left the "magic circle of play" (Salen and Zimmerman 2003). This shows how 
movement variety in mediated environments can lead to problems, but also opportunities for finding alternative ways to reach the game's goal.

We agree with Salen and Zimmerman that this aspect of rule-breaking is more likely to occur in exertion games; they attribute it to the "athletic nature" of the game (Salen and Zimmerman 2003). Salen and Zimmerman compare this effect to a chess game, in which a player will not gain an advantage by having a little corner of his/her rook peek into an adjacent square. "But in the infinitely granular space of the real world, milliseconds and millimeters can mean the difference between winning and losing" (Salen and Zimmerman 2003).

Movement variety supports creativity through finding alternative strategiesincluding cheating - to play, including the exploitation of technological limitations. This can facilitate social play ranging from disclosing to showcasing these alternative strategies.

\subsection{Uncertainty}

Table Tennis for Three exploits the affordances of tangible objects, which includes the ball, bat, and table. For our players, the tangibility of the play objects contributed to an uncertainty of play, creating opportunities for excitement and surprise. These opportunities for excitement and surprise appear to have fueled social interactions between players. The notion of surprise has been previously recognized in physical play (Czajkowski n.d.) and augmented mixed reality games (Sharp et al. 2007). The results of uncertainty contribute to Gaver's claim that the physical environment can provide affordances for social interaction in games (1996), and Hornecker and Buur's suggestion that "the richness of bodily movement" in combination with tangible interfaces is particularly beneficial for social interactions (2006). This also supported the leveling of the playing field between participants of different athletic abilities: a player who was behind in points could all of a sudden receive an advantage due to a surprise event, adding to the excitement of the game. Exertion amplified the chances and outcomes of tangibility's uncertainty: tangibility can support uncertainty without exertion; however, the diverse, fast, and forceful movements exhibited in exertion play facilitated these surprising moments for the players. Also, involving the body and the "real world" has been pointed out to add an element of uncertainty in location-based mobile phone games, which designers need to be aware of (Benford et al. 2003).

In non-exertion digital game play, these chance encounters need to be artificially introduced as an element of chance is inherent in most computer games. Game creators have to take special care in finding a balance between believable chance and randomness for the players (Salen and Zimmerman 2003). For example, in an exertion game such as the Nintendo Wii Sports Tennis, the ball on the screen might also be controlled by an element of chance; but it will be generic, as the ball will never bounce off the furniture that surrounds the player. The ball 
will also not bounce off the racquet's frame in much unexpected ways, but if it does, the experience will be "fundamentally different," as players might not believe the probability by which it occurred, but rather assume a bug in the software (Gaver 1996).

Utilizing the uncertainty that arises in physical exertion play, in particular when the body interacts with physical objects, can add an element of surprise that facilitates social play.

\section{Contributions}

This work has contributed toward an understanding of social and physical exertion play through the following contributions.

Shared object: Shared virtual objects can be used to support social play. However, they might facilitate non-parallel play in the virtual world, whereas the exertion activity can be parallel in the physical world.

Anticipation: Supporting the continuousness of bodily movements allows anticipating a player's next action, contributing to social play.

Secondary performance: Supporting people in expressing themselves using their bodies - in and outside the game-can contribute to social play and facilitate metagaming.

Movement variety: Movement variety supports creativity through finding alternative strategies - including cheating - to play, including the exploitation of technological limitations. This can facilitate social play ranging from disclosing to showcasing these alternative strategies.

Uncertainty: Utilizing the uncertainty that arises in physical exertion play, in particular when the body interacts with physical objects, can add an element of surprise that facilitates social play.

\section{Limitations}

We made the assumption that the user data gathered in a lab environment is representative to data that would have been collected in the field. Prior research has investigated the use of exertion games in people's homes in order to investigate the impact of their day-to-day surroundings on the experience, such as the limited space issues when playing Dance Dance Revolution (Sall and Grinter 2007). The authors found that the living room is an unfamiliar and often unsuitable space for physical exercise, hence the lab environment might be just as suitable to investigate an exertion game. Furthermore, we have tried to set up the table tennis tables in ways that resembles spaces in which such tables could be encountered, for example public areas in corporate environments. Also, traditional exercise is usually performed in a gym or on an outdoor field to which the participants 
generally need to travel to, just as they had to travel to participate in a game of Table Tennis for Three. There are also dedicated spaces in which commercial entities offer exertion game experiences (XRtainment), sometimes described as interactive gyms, and these places also require participants to leave their familiar surroundings and travel first before they can participate. These examples demonstrate that a dedicated environment for conducting the study that is unfamiliar for the participants is not very unusual in the context of exertion games.

The participants were located in different parts of the building. This is not the same as being in different locations across the world, separated by significant distance that requires effort to overcome by travel. As the participants knew they were able to join one another physically after the game with ease, their social behavior might have been different than if they would have been geographically very far apart. The social implications that come with simulated distance have been put aside in this study.

It is also acknowledged that scaling the system from a two-player version to a three-player system is only offering limited opportunities for investigating scaling effects of mediated social play. Online computer games have pushed the envelope of how massive scaling can be supported in gaming, with some titles supporting millions of players. Supporting three players in Table Tennis for Three seems meager in comparison. However, the system only served as vehicle to investigate social play beyond two players, and represents the first attempt to support player constellations that are otherwise hard to achieve in traditional settings: allowing three players to play together equally while investing physical effort.

\section{Future Work}

Similar to other studies who identified a performative aspect afforded by physical exertion play including audience participation that entices social play, we also observed how physical exertion play can "turn the body into a spectacle" (Sheridan and Bryan-Kinns 2008). Our current work on Table Tennis for Three did not include the consideration of an audience aspect. Therefore, we recommend future work to investigate an audience's role in social and physical exertion play, furthering our understanding of such play experiences.

Other possible avenues for future work are investigations into the applicability of the findings in co-located experiences. Furthermore, exploring how useful the themes are for describing other play interactions and analyzing existing games might also be beneficial future work. In particular, the notion of secondary performance has been investigated in other research work on social and physical interaction around games (Segura et al. 2013) since presented in this article, as such, an exploration of the themes' potential to influence new game ideas might also be a fruitful area for future work. 


\section{Conclusion}

We have presented a qualitative analysis of player observations and interviews from an exertion game to understand the facilitating role of design in the interrelationship between social and physical exertion play. First, we have found evidence that exertion games can facilitate social play, even in mediated environments. Second, we have identified the salient themes Shared Object, Anticipation, Secondary Performance, Movement Variety, and Uncertainty that contribute to the link between social and physical exertion play and discussed how specific design elements can facilitate (and hinder) this link. Our hope is that these themes are used to analyze existing as well as create future physical exertion games. Furthermore, we hope our work also has implications for theory that articulates the interrelationship between social and physical exertion play. As such, our work might also offer guidance for future work that aims to include exertion aspects into social play as well as for exertion games that are currently not supporting social play. However, we also note that design alone cannot guarantee social play, it is after all the players who create social play, that design features can only facilitate (Salen and Zimmerman 2003).

In sum, our goal is to contribute to a better understanding of social and physical exertion play and their interrelationship, advancing this research area featured within this book, in order to facilitate the many benefits of social and physical exertion play.

Acknowledgments The authors wish to thank everyone who has helped with the project and the writing of this article. The first author would also like to thank the University of Melbourne, with which he was affiliated while the majority of this work was done.

\section{References}

Bekker T, Sturm J, Eggen B (2010) Designing playful interactions for social interaction and physical play. Pers Ubiquit Comput 14(5):385-396. doi:10.1007/s00779-009-0264-1

Benford S, Anastasi R, Flintham M, Drozd A, Crabtree A, Greenhalgh C, Tandavanitj N, Adams M, Row-Farr J (2003) Coping with uncertainty in a location-based game. IEEE Pervas Comput 2(3):34-41

Benford S, Schnädelbach H, Koleva B, Anastasi R, Greenhalgh C, Rodden T, Green J, Ghali A, Pridmore T, Gaver B (2005) Expected, sensed, and desired: a framework for designing sensing-based interaction. ACM Trans Comput Hum Interact (TOCHI) 12(1):3-30

Bikeboard (n.d.). http://nyx.at/bikeboard/Board/showthread.php?threadid=61242

Consalvo M (2007) Cheating: gaining advantage in videogames. The MIT Press, Cambridge

Consolvo S, Everitt K, Smith I, Landay JA (2006) Design requirements for technologies that encourage physical activity. Paper presented at the proceedings of the SIGCHI conference on human factors in computing systems, Montreal

Consolvo S, Klasnja P, McDonald DW, Avrahami D, Froehlich J, LeGrand L, Libby R, Mosher K, Landay JA (2008) Flowers or a robot army?: encouraging awareness and activity with 
personal, mobile displays. Paper presented at the proceedings of the 10th international conference on Ubiquitous computing, Seoul

Czajkowski Z (n.d.) The essence and importance of timing (sense of surprise) in fencing. http:// www.mat-fencing.com/Akademia16.html

de Kort YAW, IJsselsteijn WA (2008) People, places, and play: player experience in a sociospatial context. Comput Entertain(CIE) 6(2)

Dourish P (2001) Where the action is: the foundations of embodied interaction. MIT Press, Boston

Fogtmann MH, Fritsch J, Kortbek KJ (2008) Kinesthetic Interaction-revealing the Bodily Potential in Interaction Design. Paper presented at the OZCHI '08 conference of the computer-human interaction special interest group (CHISIG) of Australia on computer-human interaction, Cairns

Gaver WW (1996) Affordances for interaction: the social is material for design. Ecol Psychol $8(2): 111-129$

Griffin S (2005) Push. Play: an examination of the gameplay button. Paper presented at the proceedings of DiGRA 2005 conference: changing views-worlds in play, Vancouver

Hornecker E, Buur J (2006) Getting a grip on tangible interaction: a framework on physical space and social interaction. Paper presented at the proceedings of the SIGCHI conference on human factors in computing systems, Montreal

Ishii H, Wisneski C, Orbanes J, Chun B, Paradiso J (1999) PingPongPlus: design of an athletictangible interface for computer-supported cooperative play. Paper presented at the SIGCHI conference on human factors in computing systems

Iso-Ahola SE, Hatfield BD (1986) Psychology of sports: a social psychological approach. Wm. C. Brown Publishers, Dubuque

Knoerlein B, Székely G, Harders M (2007) Visuo-haptic collaborative augmented reality pingpong. In: International conference on advances in computer entertainment technology, 2007, pp 91-94, ACM Press, New York

Larssen A, Loke L, Robertson T, Edwards J, Sydney A (2004) Understanding movement as input for interaction-a study of two eyetoy games. Paper presented at the proceedings of OzCHI ' 04 , Wollongong

Lawn M, Takeda $T$ (1998) Design of an action interface with networking ability for rehabilitation. In: IEEE Engineering in Medicine and Biology Society, Hong Kong, 1998

Lin J, Mamykina L, Lindtner S, Delajoux G, Strub H (2006) Fish'n'Steps: encouraging physical activity with an interactive computer game. Paper presented at the UbiComp 2006: ubiquitous computing conference

Lindley SE, Le Couteur J, Berthouze NL (2008) Stirring up experience through movement in game play: effects on engagement and social behaviour. Paper presented at the proceeding of the twenty-sixth annual SIGCHI conference on human factors in computing systems, Florence

Linehan C, Roche B, McLoone S, Ward T (2006) Network latency in on-line gaming: an engineering or a psychological problem? Paper presented at the CGAMES 2006-9th international conference on computer games: AI, animation, mobile, educational and serious games, Dublin Institute of Technology, Dublin

McCarthy J, Wright P (2004) Technology as experience. The MIT Press, Boston

Merleau-Ponty M (1945) Phenomenology of perception (Routledge Classics). Routledge, New York

Moen J (2006) KinAesthetic movement interaction: designing for the pleasure of motion. Dissertation, KTH, Numerical Analysis and Computer Science, Stockholm

Mueller F, Agamanolis S, Picard R (2003) Exertion interfaces: sports over a distance for social bonding and fun. Paper presented at the SIGCHI conference on human factors in computing systems, Ft. Lauderdale

Mueller F, Edge D, Vetere F, Gibbs MR, Agamanolis S, Bongers B, Sheridan JG (2011) Designing sports: a framework for exertion games. Paper presented at the CHI '11: proceedings of the SIGCHI conference on human factors in computing systems, Vancouver 
Mueller F, Gibbs M, Vetere F (2008a) Taxonomy of exertion games. Paper presented at the OzCHI '08: proceedings of the 20th Australasian conference on computer-human interaction, Cairns

Mueller F, Gibbs M, Vetere F (2009) Design influence on social play in distributed exertion games. Paper presented at the CHI '09: proceedings of the SIGCHI conference on human factors in computing systems., Boston

Mueller F, Gibbs M, Vetere F, Agamanolis S (2008b) Design space of networked exertion games demonstrated by a three-way physical game based on Table Tennis. Comput Entertain 6(3):1-31. doi:http://doi.acm.org/10.1145/1394021.1394029

Mueller F, Gibbs MR, Vetere F (2010) An exploration of exertion in mixed reality systems via the "Table Tennis for Three" game. In: Dubois E, Gray P, Nigay L (eds) Engineering of mixed reality systems. pp 165-182

Namco (2010) Tekken. Namco. http://tekken.com

Rettie R (2003) Connectedness, awareness and social presence. Paper presented at the presence 2003, 6th annual international workshop on presence, Aalborg

Salen K, Zimmerman E (2003) Rules of play: game design fundamentals. The MIT Press, Boston

Sall A, Grinter RE (2007) Let's get physical! in, out and around the gaming circle of physical gaming at home. Comput Support Co-op Work (CSCW) 16(1):199-229

Segura EM, Waern A, Moen J, Johansson C (2013) The design space of body games: technological, physical, and social design. Paper presented at the proceedings of the SIGCHI conference on human factors in computing systems, Paris

Sharp H, Rogers Y, Preece J (2007) Interaction design: beyond human computer interaction. Wiley, West Sussex

Sheridan J, Bryan-Kinns N (2008) Designing for performative tangible interaction. Int J Arts Technol Special Issue Tangible Embed Interact 1(3/4):288-308

Vossen DP (2004) The nature and classification of games. Avante 10(1):53-68

Weinberg RS, Gould D (2006) Foundations of sport and exercise psychology. Human Kinetics, Champaign

Winograd T, Flores F (1986) Understanding computers and cognition: a new foundation for design. Ablex Publishing Corporation, Norwood

Woodward C, Honkamaa P, Jppinen J, Pykkimies EP (2004) Camball-augmented virtual table tennis with real rackets. Paper presented at the proceedings of the 2004 ACM SIGCHI international conference on advances in computer entertainment technology, Singapore

XRtainment (n.d.) XRtainment—where working out is all play. http://www.xrtainmentzone.com/ 